\title{
Reproduction of "Evaluating Adversarial Evasion Attacks in the Context of Wireless Communications" and "Convolutional Radio Modulation Recognition Networks"
}

\author{
Kyle W. McClintick \\ kwmcclintick@wpi.edu \\ Worcester Polytechnic Institute \\ Worcester, MA, USA
}

\author{
Alexander M. Wyglinski \\ alexw@wpi.edu \\ Worcester Polytechnic Institute \\ Worcester, MA, USA
}

\begin{abstract}
In this work, we reproduce several findings from references [1] and [2] which possess the same title. The authors of [2] trained a Convolutional Neural Network (CNN) to classify In-phase/Quadriture (IQ) signals to one of 11 possible modulation classes, while the authors of [1] performed a detailed analysis of the trade-off between disrupting an eavesdropper using the CNN from [2] and maintaining a low Bit Error Rate (BER) in a wireless transmit-receive chain.

We found that 6 out of the 11 classes presented in the data set generated by [2] experience significant amounts of multicollinearity, even in zero-noise scenarios, due in part to the relatively small size of the input signals. Additionally, the SNR is computed with an error of approximately $6 \mathrm{~dB}$ for the BPSK class, which is an amount that varies for other classes in the data set. Finally, we determine there exists a need for greater variety in the data set channels, dimensions, and oversampling to enable further investigate trade-offs.

We found the CNN used in [1] does indeed require dither to generate the BPSK signals reported due to zero-valued gradients being computed for the signals' quadrature components. Alternatively, bias terms may be used, although this significantly impairs training. The energy and SNR issues present in [2] could potentially be mentioned when utilized by [1], since there is a mismatch for both axis, which occurs when reproducing the eavesdropper classifier accuracy plots shown in [1].
\end{abstract}

Keywords: adversarial perturbations, datasets, neural networks, wireless

\footnotetext{
Permission to make digital or hard copies of all or part of this work for personal or classroom use is granted without fee provided that copies are not made or distributed for profit or commercial advantage and that copies bear this notice and the full citation on the first page. Copyrights for components of this work owned by others than ACM must be honored. Abstracting with credit is permitted. To copy otherwise, or republish, to post on servers or to redistribute to lists, requires prior specific permission and/or a fee. Request permissions from permissions@acm.org.

CPS-IoTBench2021, May 18, 2021, Nashville, TN, USA

(C) 2021 Association for Computing Machinery.

ACM ISBN 978-1-4503-8439-1/21/05 ...\$15.00

https://doi.org/10.1145/3458473.3458818
}

ACM Reference Format:

Kyle W. McClintick and Alexander M. Wyglinski. 2021. Reproduction of "Evaluating Adversarial Evasion Attacks in the Context of Wireless Communications" and "Convolutional Radio Modulation Recognition Networks". In Proceedings of CPS-IoTBench '21: A community effort to better evaluate and compare low-power wireless networks (CPS-IoTBench '21). ACM, New York, NY, USA, 6 pages.

\section{Introduction}

The Supervised Learning (SL)-based wireless communications and auxilary data set generation work presented in [3] yielded a host of publications that has piqued the interest of the SL-based wireless community, both from the original authors [2,4-7] as well as from other groups [1, 8-11]. By open sourcing the methodology for creating these data sets, any researcher possesses the capability to modify this framework it for their specific application or use. Currently, this data set is the only one of its kind within the SL-based wireless communications domain with no suitable alternatives. Given its significant contribution to the research community, it has been observed there exists a small amount of miscalculations in the generation of the data set, and there exists an opportunity to update the data set generation code to further explore trade-offs in classification and learning tasks.

Some works that makes use of these data sets craft adversarial perturbations, which are signals added to the inputs of trained SL models with the intent of causing misclassification at the testing phase [12]. Consequently, the eavesdropper will incorrectly classify the modulation class of observed Over-the-Air (OTA) signals via the transmitter computing Fast Gradient Sign Method (FGSM) adversarial perturbations in an untargeted, white box attack on the eavesdropper's trained CNN model. The perturbations are constrained, or alternatively they are optimized to have minimal norms, $\ell_{p}, p=0,1,2, \infty$, such that, depending on the threat model assumed, they are visually imperceptible, possess the lowest probability of being detected by automated or human observers, or cause the smallest number of bit errors. While there are several published analysis on digital attacks against radio systems using adversarial perturbations $[9,10]$, there exists a need for works such as [1] that investigate the effect 


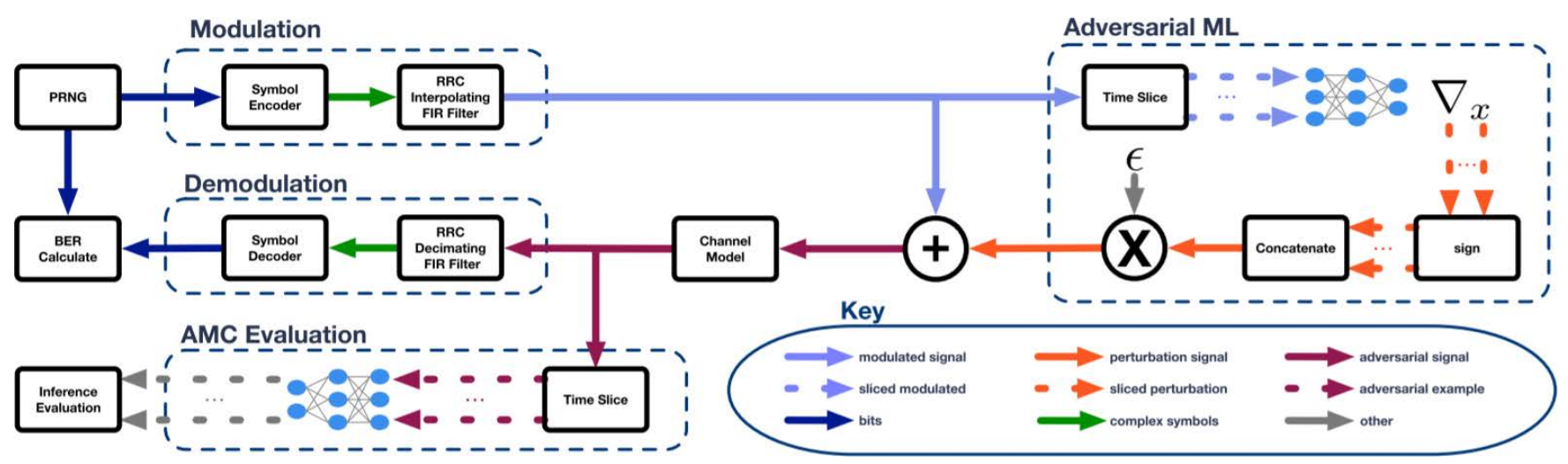

Figure 1. The threat model presented in [1], in which a transmitter aims to protect the privacy of a transmission by performing a white box attack against an eavesdropping radio equipped with a CNN-based modulation classifier.

of channel noise on the success of these minimal signals at causing SL models to misclassify.

In this reproduction work, we discuss the threat model in Section 2, and train the Convolutional Neural Network (CNN)-based eavesdropper presented in [2] using the data set generated by [3] in Section 3. We reproduce the Fast Gradient Sign Method (FGSM) adversarial perturbations presented in [1], and plot the eavesdropper classification accuracy versus Signal-to-Noise Ratio (SNR) in Section 4. Finally, we present our closing remarks in Section 5.

\section{Review of Threat Model}

In [1], it is assumed the transmitter has no knowledge of the channel such that FGSM perturbations are computed from and added to the transmitted signal before the channel (Figure 1) as:

$$
x^{*}=x+\epsilon \cdot \operatorname{sign}\left(\nabla_{x} \mathcal{L}(f(\theta, x), y)\right),
$$

where $x$ is the transmitted signal and model input, $\epsilon$ is the power constraint of the perturbation, and the FGSM perturbation is the sign of the gradient $\nabla_{x}$ of the model's prediction error with respect to the input. The prediction error is given by the categorical cross entropy loss between the true label $y$ and the predicted label, which is a function of the input $x$ and the trained model parameters $\theta$. It is assumed the receiver and the eavesdropper receive the same transmitted signal through i.i.d. realizations of the wireless channel. The wireless channel model is implemented using GNU Radio's dynamic channel model, which simulates a tap-based multipath wireless channel with Additive Gaussian White Noise (AWGN), Rician fading, and a truncated Gaussian-based random walk of the symbol rate and carrier frequency offset. The random delay and drift of the transmitting time slice block is removed in favor of a deterministic scheme to simulate the synchronization of the transmitting and receiving radios in the system. However, the eavesdropper's time slice has a small uniform random delay and drift in the sampling of each input slice. As a "white box" attack, it is assumed the transmitter has complete knowledge of the eavesdropper $\mathrm{CNN}$ trained weights and structure. The perturbations are $\ell_{2}$ power constrained by the FGSM threshold:

$$
\epsilon=\sqrt{\frac{10^{\frac{E_{p}}{E_{s}}} / 10}{2 \times I}},
$$

given symbol energy $E_{s}=\int|x|^{2} d x$, perturbation symbol energy $E_{p}=\int\left|x^{*}-x\right|^{2} d x$, and interpolation rate $I=8$ which is fixed and known by the transmitter and the constrained perturbation to signal ratio chosen by the operator $-\infty<$ $E_{p} / E_{s}<0$.

\section{Analysis of the Trained Eavesdropper}

In order to reproduce the FGSM perturbations in [1], we first trained the CNN model as described in [13]. The CNNbased eavesdropper from [13] differs from the original CNN developed by the authors one year prior in [2] by using length 7 filters instead of length 3, and by using no bias terms in the first convolutional layer. The model estimates 11 modulation class scores for input signals of 128 IQ samples by using two convolutional layers, a dense layer, and a softmax layer.

Signals with SNR values equal to $18 \mathrm{~dB}$ produced a significant variance in the classification accuracy for each class. Of the 11 classes, 5 have nearly $100 \%$ classification accuracy, while the other 6 have as low as $50 \%$ accuracy (Figure 2). We seek to explain these incorrect classifications as being a consequence of multicollinearity, i.e., the similarities between inputs of different classes.

A visual inspection of the data set reveals that three pairs of classes share a subset of common constellation points (Figure 3). Further, it was found that many of the 128-sample (16 symbols at 8 SPS) IQ signals serving as inputs to the $\mathrm{CNN}$ model do not include every equiprobable symbol, due to their small size and high interpolation rate of $I=8$. As an example, the modulation class QPSK has an order of $M=4$ 
Reproduction of "Evaluating Adversarial Evasion Attacks in the Context of Wireless Communications" and "Convolutional Radio Modulation Recognition Networks"

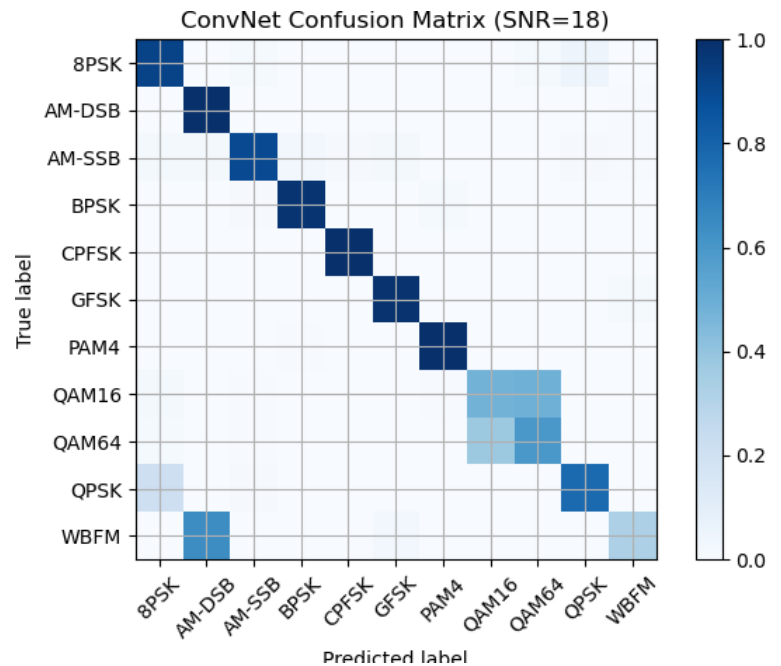

Figure 2. The test-set confusion matrix for signals with $\mathrm{SNR}=18 \mathrm{~dB}$. Even at high SNR, three pairs of modulation classes produce incorrect classifications, potentially due to multicollinearity.

representing bit code words $\in\{00,01,10,11\}$. A typical 16symbol sequence would contain four of each symbol, but several signals in the data set may contain only samples produced by the symbols $\in\{00,01,11\}$ without any " 10 " symbols. This issue is especially noticeable in higher order schemes such as QAM16 and QAM64. Consequently, these low-noise misclassifications are caused by signals whose samples fall near the shared, ambiguous constellation points with its most similar class.

This multicollinearity alerts us to a fundamental trade-off between latency and accuracy. Larger time-slices will lead to lower multicollinearity but increase the delay before a prediction can be made by the trained CNN model. A data set that includes a variation of input data dimensions would allow for further research that may yield insight into this trade-off. However, this would require additional pre-processing or a change of models to a Recurrent Neural Network (RNN) or some other predictor that allows for varying input dimensions.

\section{FGSM Perturbations}

The FGSM perturbations are produced in Python using TensorFlow and the GNU Radio Python Module. A GNU Radio top block is created in a Python script, and the FGSM perturbations are computed using the trained model and TensorFlow to compute the gradient of the model's class scores with respect to the pre-channel signal.

We found the CNN described in [1] did require a dither in order to produce the pre-channel BPSK signal and perturbation they reported (Figure 4). Without the dither, the lack of a bias term in the first convolutional layer of the modulation

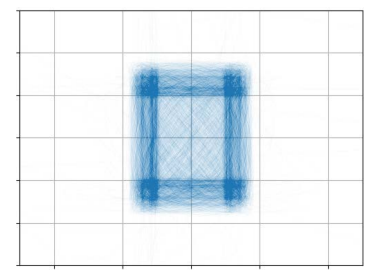

(a) QPSK

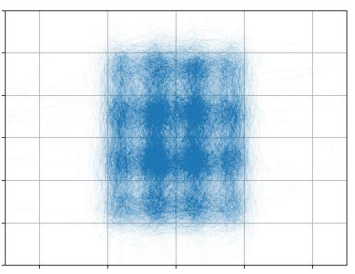

(c) QAM16

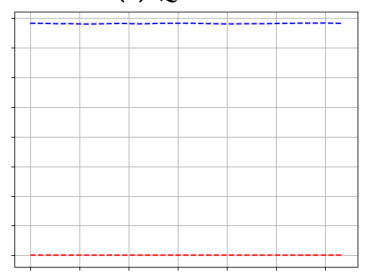

(e) AM-DSB

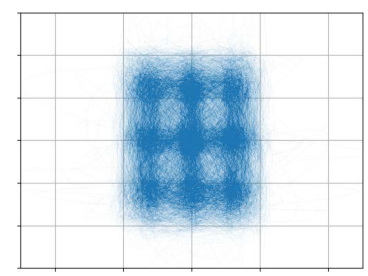

(b) 8 PSK
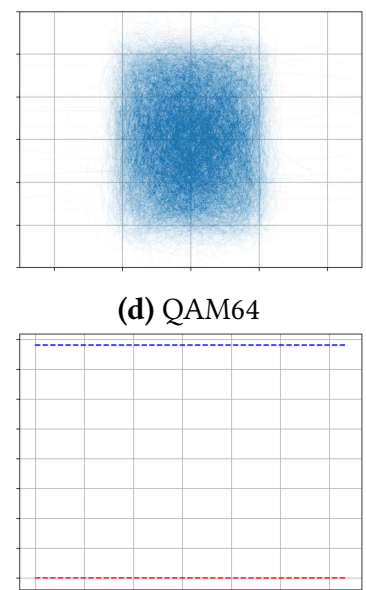

(f) WBFM (d) QAM64

Figure 3. A visual inspection of every $\mathrm{SNR}=18 \mathrm{~dB}$ IQ sample set for the 6 signal classes in the RML2016.10a data set [3], that produced significant misclassifications due to multicollinearity (Figure 2).

classifier caused the zero-valued quadrature samples of the BPSK signals to produce zero-valued class scores and consequently zero-valued gradients with respect to the model input. Since the sign function from equation (1) is:

$$
\operatorname{sign}(x)= \begin{cases}-1 & x<0 \\ 0 & x=0 \\ 1 & x>0\end{cases}
$$

a zero-valued gradient will produce zero-valued perturbations. While including a bias term in the first convolutional layer fixes this issue, we found this solution produced a vanishing gradient during training, which agrees with the findings of [1].

In [1], the authors equate the energy per symbol and SNR, $E_{S} / N_{0}=S N R$, where $E_{S}=1$ is enforced as a pre-channel step, Gaussian noise is generated with variance:

$$
\sigma_{[1]}=\sqrt{10^{-S N R / 10 / 2}}
$$

and SNR is chosen. In [2], Gaussian noise is generated with a variance of:

$$
\sigma_{[2]}=10^{-S N R / 10},
$$

and each post-channel, 128-sample noisy signal is powernormalized. In checking both works actual SNR for BPSK 


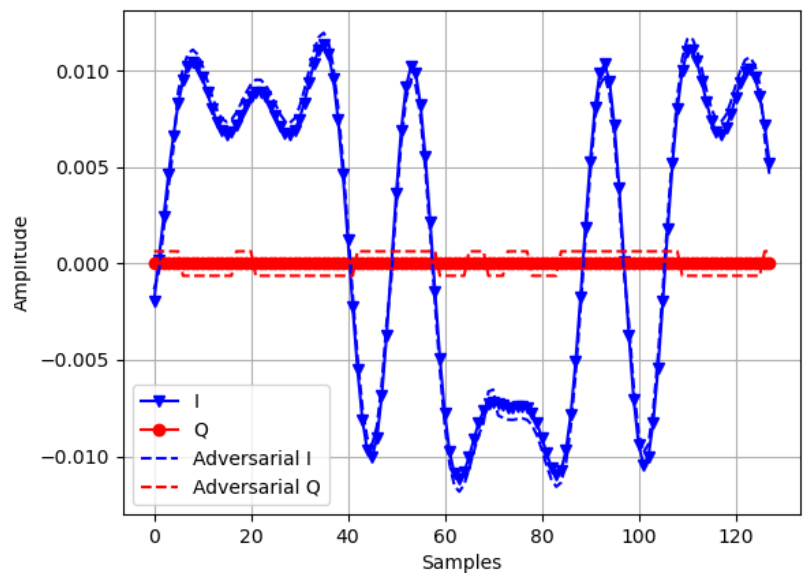

(a) $E_{p} / E_{s}=-10 d B$ signal with $\mathrm{CNN}$ bias term obtained by following [2].

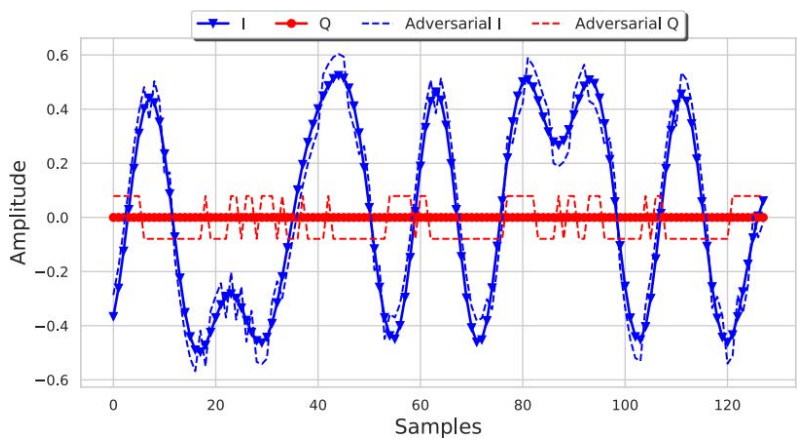

(b) Reported $E_{p} / E_{s}=-10 d B$ signal from [1] without CNN bias term.

Figure 4. The quadrature samples of the perturbation cannot be non-zero unless a bias term is used in the first convolutional layer of the CNN, or dither is introduced to signals as a pre-processing step. Additionally, the signal shown in (a) is power normalized post-channel $\left(16 \times E_{s}+N_{0}=1\right)$, whereas each symbol of the signal shown in $(\mathrm{b})$ is power normalized $\left(E_{s}=1\right)$. Finally, the signal shown in (a) incorrectly computes $\epsilon$ because $E_{s} \neq 1$, which is a key assumption of equation (2).

signals as:

$$
\mathrm{SNR}=10 \log _{10}\left(I \times P_{s} / P_{n}\right),
$$

we found that [1] correctly generates noise with their variance, where as [2] has an offset of approximately $6 \mathrm{~dB}$, such that signals described as having an SNR of $0 \mathrm{~dB}$ are in reality possessing an SNR of $6 \mathrm{~dB}$ (Figure 5). This presents an issue in reproducing work, as noise is being described as possessing a higher power level than it actually does, causing classification performance to seem better than it should be at any given SNR value.

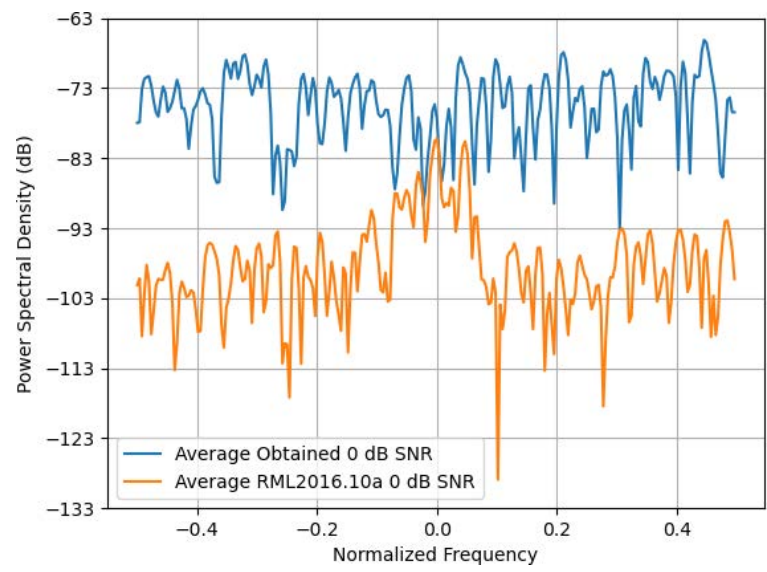

Figure 5. The averaged frequency content of all reported $\mathrm{SNR}=0 \mathrm{~dB}$ signals from the BPSK class of the RML2016.10a data set. Additionally, we computed the average spectral power of signals generated from the same software, but with the AWGN added separately using our own computation.
A consequence of the non-unit signal energy present by signals generated in [2] causes the application of equation (2) to compute perturbations that are too small, and potentially less effective than the larger FGSM attacks generated by [1] (Figure 6).

As in the reproduced work, we found the perturbations did not impact the performance of the classifier in high noise channels since all the signals will be statistically dominated by the AWGN distribution and the classifier cannot differentiate the signals with or without perturbations. Additionally, we found that as $E_{p} / E_{s}>E_{p} / N_{0}$, the FGSM perturbations become more effective since the channel noise impairs both the classifier and the FGSM perturbations (Figure 6).

\section{Discussion}

Overall, we found the description of the data set, CNN model, and computation of FGSM perturbations in [1] sufficient to reproduce the work, and agree with all findings found therein.

We observe the existence of several issues with the underlying, open source data set utilized by [1], such as the SNR of the noisy signals generated by the data set originally from [2] is approximately $6 \mathrm{~dB}$ higher than reported, and the symbol energy is not normalized $\left(E_{s}=8\right)$ for ease of perturbation or noise ratios. Consequently, scaling and axis mismatch occurs when computing the perturbation scaling and resulting eavesdropper classification accuracy given by the threat model in [1]. Flowers et al. present an open-source tutorial for reproducing their work despite both of these mismatches in the code available at github.com/brysef $/ \mathrm{rml}$. 
Reproduction of "Evaluating Adversarial Evasion Attacks in the Context of Wireless Communications" and "Convolutional Radio Modulation Recognition Networks"
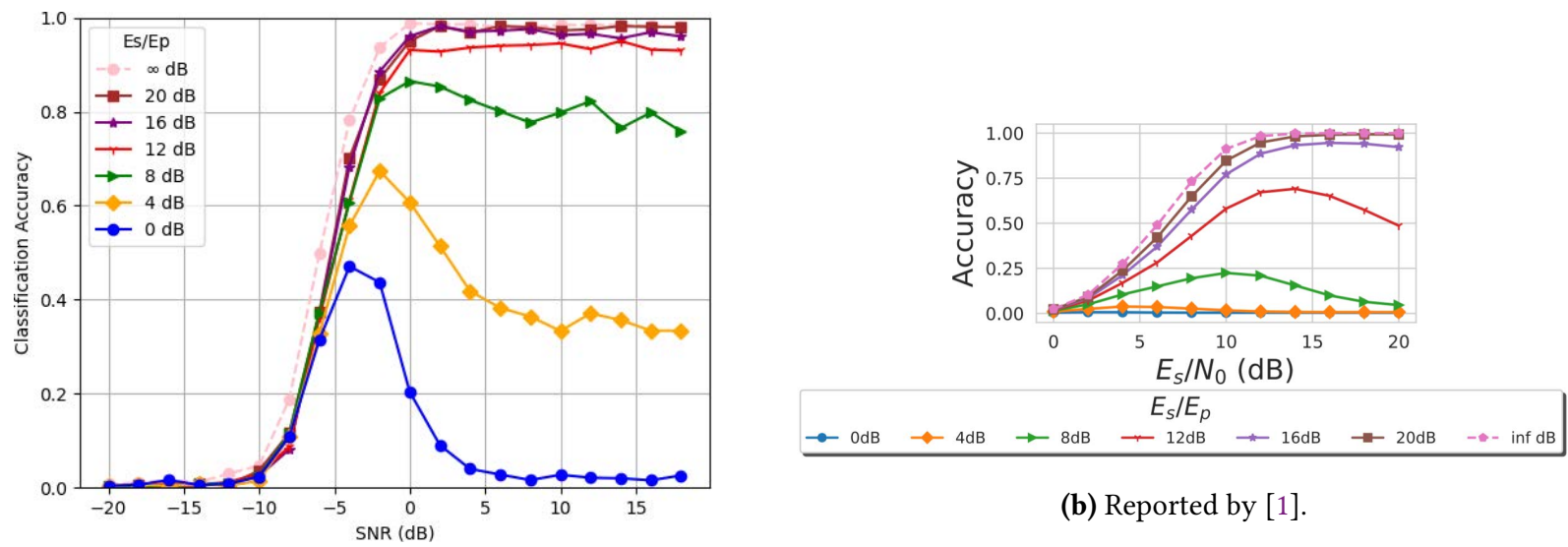

(b) Reported by [1].

(a) CNN accuracy during FGSM attacks obtained by following [2].

Figure 6. Classification accuracy of the trained CNN-based eavesdropper for a fixed power BPSK signal with varying signal to noise and signal to perturbation ratios. The SNR axis shown in figure (a) is shifted by approximately $-6 \mathrm{~dB}$ compared to figure (b). Additionally, the symbol energy normalization mismatch causes the perturbations to be smaller than reported by the legend in figure (b).

Six of the eleven modulation classes of the data set generated in [2] were seen to experience multicollinearity issues in even noiseless scenarios. Consequently, there exists a need for a data set that offers a wider range of channels, input dimensions, and over-sampled rates to allow investigations into multi-dimensional trade-offs. With just one, highly specific dynamic channel model, channel effects cannot be isolated to diagnose the cause of CNN misclassifications. Without varying input dimensions, latency and multicollineraity trade-offs cannot be investigated. Finally, the high oversampling present (8 SPS) is overly optimistic for most applied radio systems that are not performing fine-grain estimation that would require significant oversampling.

While the RML2016.10a data set was originally intended for modulation classification, the community has used it extensively in the application of adversarial perturbations, which requires the computation of BER to investigate security, power, and communication loss trade-offs. Consequently, there exists a need for the adaptation of the RML2016.10a data set or the development of a new data set that meets this need for simulating an example receiver chain. This open source code should be adaptable and implement relevant receiver aspects such as filtering, which removes out of band noise and perturbations, amplifiers, which clip large amplitude perturbations due to non-linearity, and bit estimation, an essential metric for method evaluation.

\section{Acknowledgements}

We would like to acknowledge William Headley and Bryse Flowers of [1], who have provided feedback and directions towards additional works and resources.

\section{References}

[1] B. Flowers, R. M. Buehrer, and W. C. Headley, "Evaluating adversarial evasion attacks in the context of wireless communications," 2019.

[2] T. J. O'Shea, J. Corgan, and T. C. Clancy, "Convolutional radio modulation recognition networks," arXiv preprint arXiv:1602.04105, 2016.

[3] T. J. O'Shea and N. West, "Radio machine learning dataset generation with gnu radio," Proceedings of the 6th GNU Radio Conference, 2016.

[4] T. J. O'Shea, T. Roy, and T. C. Clancy, "Over-the-air deep learning based radio signal classification," IEEE Journal of Selected Topics in Signal Processing, vol. 12, no. 1, pp. 168-179, 2018.

[5] T. J. O'Shea, T. Roy, and T. C. Clancy, "Over the air deep learning based radio signal classification," CoRR, vol. abs/1712.04578, 2017.

[6] T. J. O'Shea, N. West, M. Vondal, and T. C. Clancy, "Semi-supervised radio signal identification," 2017.

[7] N. E. West and T. J. O'Shea, "Deep architectures for modulation recognition," 2017.

[8] B. Flowers, R. M. Buehrer, and W. C. Headley, "Communications aware adversarial residual networks for over the air evasion attacks," in MILCOM 2019 - 2019 IEEE Military Communications Conference (MILCOM), pp. 133-140, 2019.

[9] M. Sadeghi and E. G. Larsson, "Adversarial attacks on deep-learning based radio signal classification,” CoRR, vol. abs/1808.07713, 2018.

[10] B. Kim, Y. E. Sagduyu, K. Davaslioglu, T. Erpek, and S. Ulukus, "Overthe-air adversarial attacks on deep learning based modulation classifier over wireless channels," 2020.

[11] M. DelVecchio, V. Arndorfer, and W. C. Headley, "Investigating a spectral deception loss metric for training machine learning-based evasion attacks," Proceedings of the 2nd ACM Workshop on Wireless Security and Machine Learning, Jul 2020.

[12] X. Yuan, P. He, Q. Zhu, and X. Li, "Adversarial examples: Attacks and defenses for deep learning," 2018.

[13] T. J. O'Shea and J. Hoydis, "An introduction to machine learning communications systems," CoRR, vol. abs/1702.00832, 2017. 